\title{
Preparação e Caracterização de Membranas Assimétricas de Poli (Fluoreto de Vinilideno) Suportadas em Poliéster - I: Efeito do Tratamento Térmico nas Propriedades das Membranas
}

\author{
Mônica B. Thürmer, Patrícia Poletto, Marcos Marcolin, Daiane G. Ferreira, Mara Zeni \\ Centro de Ciências Exatas e Tecnologia, UCS
}

\begin{abstract}
Resumo: O poli(fluoreto de vinilideno) (PVDF) é um polímero semicristalino contendo uma fase amorfa e uma cristalina. Essa característica é importante para o preparo de membranas assimétricas, pois a fase cristalina tem grande influência na formação da estrutura da membrana e a fase amorfa na porosidade. Membranas de PVDF foram preparadas pelo processo de inversão de fases e avaliadas quanto à permeabilidade e morfologia, bem como o efeito do tratamento térmico. Foram comparadas as seguintes membranas: membrana sem tratamento térmico, membrana com tratamento térmico e membrana comercial. Ensaios de resistência química, fluxo permeado de água a várias pressões, difusão de íons, Microscopia Eletrônica de Varredura (MEV), Calorimetria Exploratória Diferencial (DSC) e Análise Termogravimétrica (TGA) foram realizados para verificar o comportamento das membranas.
\end{abstract}

Palavras-chave: $P V D F$, tratamento térmico, inversão de fases, membrana polimérica.

\section{Preparation and Characterization of Asymmetric Membranes of Poly (Vinylidene Fluoride) Supported in Polyester - I: Effect of Heat Treatment in the Properties of Membranes}

\begin{abstract}
Poly(Vinylidene Fluoride) (PVDF) is a semicrystalline polymer containing an amorphous and a crystalline phase. This characteristic is important for the preparation of asymmetric membranes, because the crystalline phase has great influence on the membranes structure, while the amorphous phase affects the porosity. PVDF membranes were prepared by the phase inversion process, which were then evaluated with regard to permeability and morphology, as well as the thermal treatment effect. The following membranes were compared: without thermal treatment, with thermal treatment and commercially-available membrane. The analysis of chemical resistance, water permeability flux to different pressures and diffusion of ions, in addition to studies with Scanning Electron Microscopy (SEM), Differential Scanning Calorimetry (DSC) and Thermogravimetric Analysis (TGA), were performed to investigate the membranes properties.
\end{abstract}

Keywords: PVDF, thermal treatment, phase inversion, polymeric membranes.

\section{Introdução}

Os Processos de Separação por Membranas (PSM) têm sido aplicados no fracionamento de misturas, soluções e suspensões envolvendo espécies de tamanho e natureza química diferentes. Dependendo da aplicação, as membranas podem apresentar diferenças significativas em termos funcionais e morfológicos. $\mathrm{O}$ conhecimento da morfologia das membranas e sua relação com as propriedades de transporte são importantes para uma melhor compreensão dos fenômenos envolvidos nos problemas de $\operatorname{separação~}^{[1]}$.

Embora recentes, os processos de separação por membranas têm sido utilizados de maneira crescente em uma ampla variedade de indústrias, tais como: as químicas, farmacêuticas, têxteis, de papel e alimentícias ${ }^{[2]}$. Membranas poliméricas podem ser obtidas por vários métodos, tais como: inversão de fase, sinterização, estiramento ou gravação, para as membranas microporosas. Já as membranas densas podem ser preparadas por: evaporação do solvente, extrusão, laminação, sopro, inversão de fase (integral) ou por deposição de um filme denso em um suporte microporoso (por espalhamento, polimerização in situ ou polimerização por plasma $)^{[1]}$.

A técnica de inversão de fases, proposta por Loeb e Sourirajan em $1960^{[3]}$, permite uma grande variabilidade na morfologia, seletividade e nas propriedades de transporte de solutos através da membrana. Dependendo de fatores como natureza e composição do polímero, solvente e não solvente, presença ou não de aditivos e as condições nas quais se realizam a coagulação (temperatura do banho, tempo de imersão e espessura do filme), obtêmse membranas com diferentes morfologias, propriedades e, consequentemente, aplicações distintas ${ }^{[4]}$.

O poli (fluoreto de vinilideno) (PVDF) é um polímero semicristalino contendo fase amorfa e fase cristalina. Essa característica é muito importante para a preparação de membranas assimétricas, pois a fase cristalina tem grande influência na formação da estrutura da membrana e a amorfa na porosidade ${ }^{[5]}$. Sendo um polímero hidrofóbico, atóxico e de alta resistência térmica e química, o PVDF é tolerante a ácidos, mas seu uso é limitado a $\mathrm{pH}$ abaixo de 11. Contudo, sua maior vantagem é a alta tolerância ao cloro ${ }^{[6]}$.

O tratamento térmico de membranas é comumente realizado com o intuito de minimizar os defeitos de superfície das membranas, visando o aumento da seletividade da mesma. Neste tratamento, devido ao aumento da mobilidade segmental do polímero, ocasionado pelo aumento de energia térmica, há um aumento do número de choques entre as moléculas e da possibilidade de formar ligações intermoleculares que, irreversivelmente, reduzem o volume livre da matriz polimérica, provocando redução no tamanho de poros superficiais por contração capilar, verificada através de um aumento na seletividade e de diminuição na permeabilidade da membrana tratada ${ }^{[7]}$.

O objetivo deste trabalho foi a comparação do desempenho de membranas de PVDF produzidas em laboratório (com e sem tratamento térmico), com membrana comercial. Foram

Autor para correspondência: Mara Zeni, Centro de Ciências Exatas e Tecnologia, Universidade de Caxias do Sul, Caixa Postal 1352, CEP: 95001-970, 
desenvolvidas membranas de poli(fluoreto de vinilideno) (PVDF) suportadas em poliéster, preparadas pelo processo de inversão de fase. As membranas preparadas foram caracterizadas por: resistência química; fluxo de água permeada; difusão de íons; Microscopia Eletrônica de Varredura (MEV) e ensaios térmicos (TGA, DSC), a fim de se verificar o comportamento das membranas e sua eficiência, nos processos de separação, contribuindo para a busca de sistemas viáveis e eficientes no tratamento de águas residuárias.

\section{Experimental}

\section{Materiais}

O poli(fluoreto de vinilideno) (PVDF) com plastificante, SOLEF 1015/0078 foi fornecido pela Solvay Solexis, Inc. O solvente utilizado foi a N'N'dimetilformamida $\left(\mathrm{C}_{3} \mathrm{H}_{7} \mathrm{ON}\right)(\mathrm{DMF})$, fornecido pela Vetec, Brasil. Como não-solvente foi utilizado água destilada. O suporte macroporoso utilizado foi um tecido $100 \%$ poliéster com espessura de $180 \mu \mathrm{m}$ e gramatura 110 g.m. ${ }^{-2}$, fornecido por Notredame Com. e Imp. Para comparação com as membranas de PVDF desenvolvidas utilizou-se a membrana comercial Durapore (membrana C), fabricada pela MilliPore. Esta membrana apresenta as seguintes características: PVDF; hidrofílica; tamanho de poro $0,45 \mu \mathrm{m}$; branca e plana; aplicada para a microfiltração de soluções biológicas; fluxo de água: $17.400 \mathrm{~L} \cdot \mathrm{h}^{-1} \cdot \mathrm{m}^{-2}$; espessura $125 \mu \mathrm{m}$.

\section{Preparo das membranas}

As membranas foram preparadas pelo método de inversão de fases. A espessura das membranas foi determinada pela espessura de um fio-guia de $0,40 \mathrm{~mm}$ de diâmetro, colocado sobre o suporte de poliéster, onde a solução polimérica de PVDF em DMF foi espalhada. As membranas em estudo apresentaram espessura final de $282 \pm 4 \mu \mathrm{m}$ (A) e $276 \pm 5 \mu \mathrm{m}$ (B). Foram preparadas membranas a partir de soluções com $20 \%(\mathrm{~m} / \mathrm{v})$ de PVDF em DMF.

Dissolveu-se o polímero em DMF, sob agitação magnética a $50{ }^{\circ} \mathrm{C}$ por 24 horas. Espalhou-se uniformemente a solução sobre o suporte fixado em uma placa de vidro, com um fio-guia de $0,40 \mathrm{~mm}$. Imediatamente após o espalhamento, imergiu-se o sistema suportesolução em banho de coagulação com água destilada à $24 \pm 2{ }^{\circ} \mathrm{C}$, obtendo-se a membrana não tratada termicamente (A). Para o tratamento térmico da membrana, colocou-se a mesma entre duas placas de vidro a $60{ }^{\circ} \mathrm{C}$ por 1 hora em estufa, obtendo-se a membrana B.

\section{Caracterização das membranas}

\section{Resistência química}

Os ensaios de resistência química das membranas foram realizados em diferentes $\mathrm{pH}(1 \mathrm{a} 14)$, por 24 horas a $25^{\circ} \mathrm{C} \pm 2$, com o controle da perda de massa.

\section{Fluxo de água permeada}

As membranas, com área de $1,64 \times 10^{-3} \mathrm{~m}^{2}$, foram testadas com água em sistema de permeação, modelo MinitamTM System da Millipore, à temperatura de $20^{\circ} \mathrm{C}$, nas pressões entre 0 e $6 \mathrm{~atm}$. Os tempos de ensaio foram de 30 minutos, nas diferentes pressões. $\mathrm{O}$ fluxo de água permeada é dado pela Equação $1^{[8]}$ :

$$
J w=\frac{V}{A . \Delta T}
$$

onde Jw é o fluxo de água permeada $\left(\mathrm{L}^{\mathrm{m}} \mathrm{m}^{-2} \cdot \mathrm{h}^{-1}\right)$, V é o volume permeado (L), A é a área da membrana $\left(\mathrm{m}^{2}\right)$ e $\Delta \mathrm{T}$ é a variação do tempo (horas).

\section{Difusão de íons}

Foi utilizado uma cela acrílica de dois compartimentos separados pela membrana, tendo em um dos compartimentos água deionizada e o outro com solução $\mathrm{KCl}\left(10^{-3} \mathrm{~mol} . \mathrm{L}^{-1}\right)$. A calibração foi construída, pela medida da condutividade a várias concentrações de $\mathrm{KCl}$ e utilizada para controle da difusão do $\mathrm{KCl}$ através da membrana. A partir do declive da concentração em função do tempo e do fluxo (J), através da membrana, o coeficiente de permeabilidade (P) e o coeficiente de difusão (D) foram determinados a partir das Equações 2 e $3^{[9]}$ :

$$
\begin{aligned}
& P=\frac{J}{\Delta C} \\
& D=P x d
\end{aligned}
$$

onde $\Delta \mathrm{C}$ é a diferença de concentração entre os dois compartimentos e d é a espessura da membrana.

\section{Análise morfológica por Microscopia Eletrônica de Varredura (MEV)}

Foram realizadas análises da superfície e da seção transversal das membranas. Para a observação da seção transversal, as membranas foram cuidadosamente fraturadas em nitrogênio líquido e metalizadas com uma fina camada de ouro. Foi utilizado um microscópio eletrônico de varredura da marca Shimadzu, modelo SSX-550.

\section{Calorimetria Exploratória Diferencial (DSC)}

As análises de DSC do suporte, do pellet de PVDF, das membranas preparadas e da membrana comercial, foram realizadas em equipamento da marca Shimadzu, modelo DSC-50. A taxa de aquecimento e resfriamento utilizada durante a análise foi de $10{ }^{\circ} \mathrm{C} / \mathrm{min}$, em atmosfera de nitrogênio a uma taxa de $50 \mathrm{~mL} / \mathrm{min}$. A temperatura variou de -40 a $300{ }^{\circ} \mathrm{C}$.

\section{Análise Termogravimétrica (TGA)}

Foram realizadas análises de TGA do suporte, do pellet de PVDF, das membranas preparadas e da membrana comercial, em equipamento da marca Shimadzu, modelo TGA-50. A taxa de aquecimento utilizada durante a análise foi de $10{ }^{\circ} \mathrm{C} / \mathrm{min}$, em atmosfera de nitrogênio, partindo de temperatura ambiente até $900^{\circ} \mathrm{C}$.

\section{Resultados e Discussão}

Os resultados obtidos pelas membranas de PVDF sem tratamento (A) e com tratamento térmico (B) foram comparados com os dados da membrana comercial (C).

\section{Resistência química das membranas}

Os testes realizados com as membranas numa faixa de pH de 1 a 14, mostraram que as mesmas são resistentes à ácidos, porém seu uso é limitado em ambientes básicos até $\mathrm{pH} 11$, conforme já descrito na literatura ${ }^{[6]}$. A perda de massa máxima para as membranas foi de $1 \%$ acima de $\mathrm{pH} 11$, onde também se constatou uma pequena alteração na cor das membranas.

\section{Fluxo de água permeada}

$\mathrm{O}$ efeito do tratamento térmico no fluxo de água pura para membranas A e B, foi investigado e é apresentado na Figura 1.

Observou-se que o fluxo médio de água permeado, para pressões menores do que 3 bar, é igual para as membranas A e B. Para pressões entre 4 e 6 bar, o fluxo médio de água permeado é maior para a membrana B. A membrana com tratamento térmico apresentou fluxo de água permeado superior ao da membrana sem tratamento. Este efeito é contrário ao esperado, já que segundo a literatura ${ }^{[7]} \mathrm{o}$ tratamento térmico ocasionaria uma redução na permeabilidade das membranas, devido ao aumento da mobilidade segmental do polímero, levando a uma contração da membrana tratada pela redução do volume livre da 


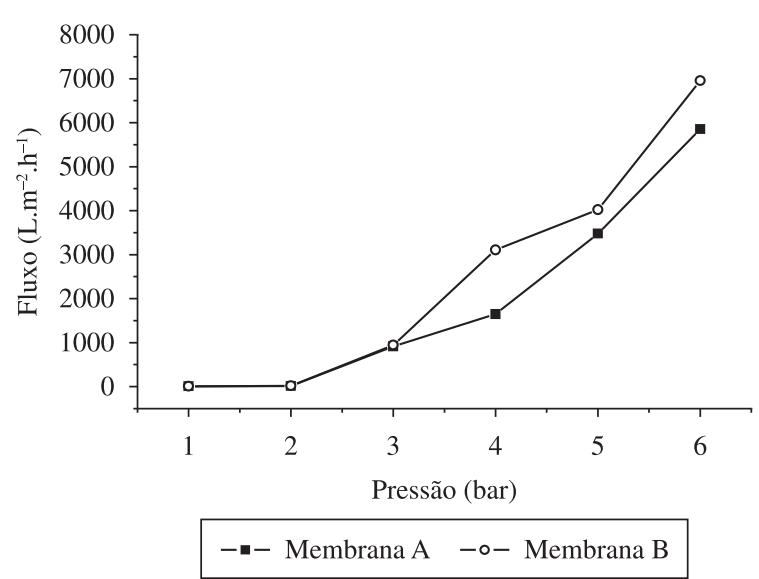

Figura 1. Fluxo permeado de água, a diferentes pressões $\left(20^{\circ} \mathrm{C}, 30\right.$ minutos $)$, para as membranas de PVDF (A e B).

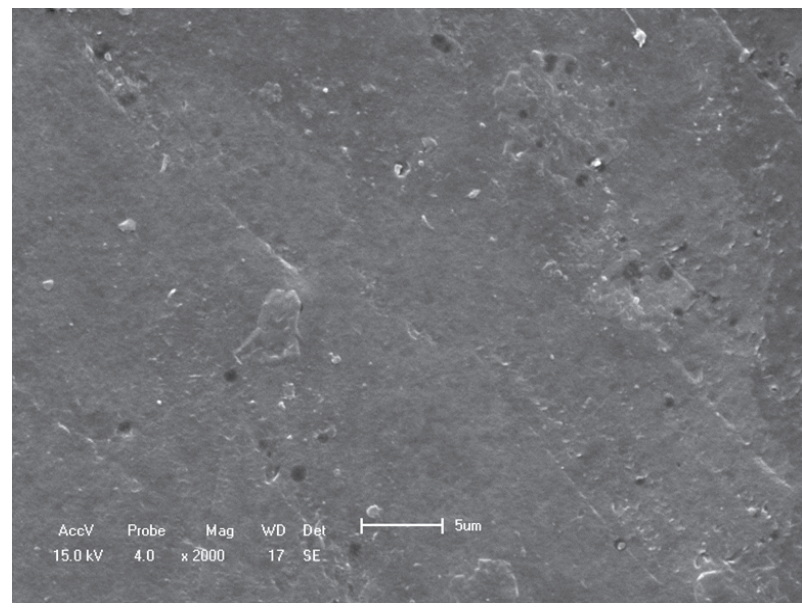

(a)

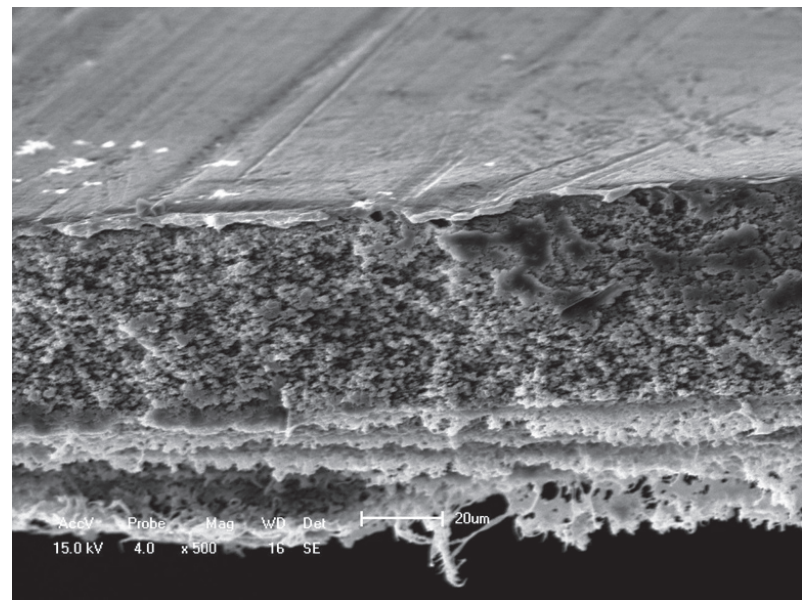

(c)

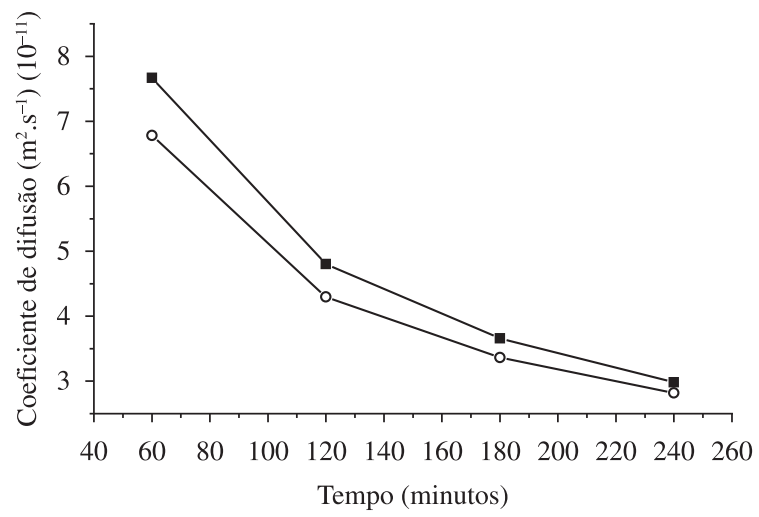

-1- Membrana A -o- Membrana B

Figura 2. Variação do coeficiente de difusão de íons com o tempo, para as membranas PVDF (A e B).

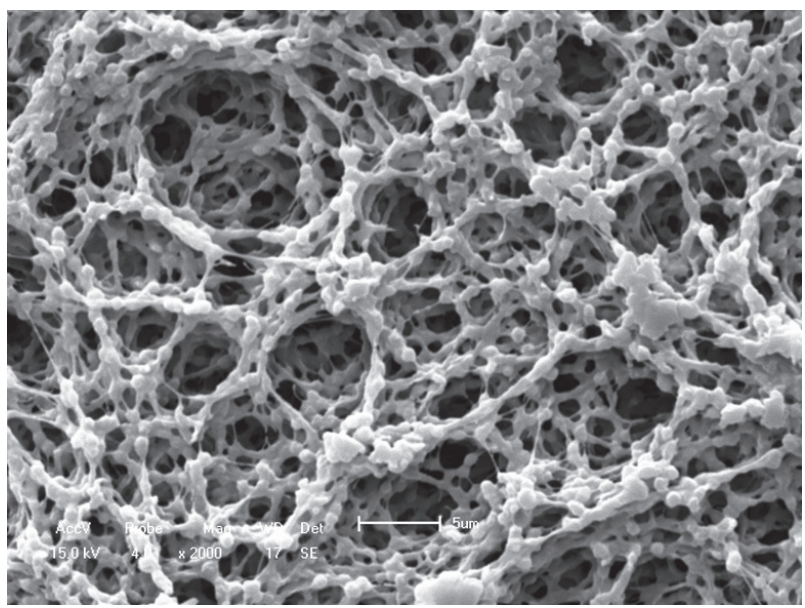

(b)

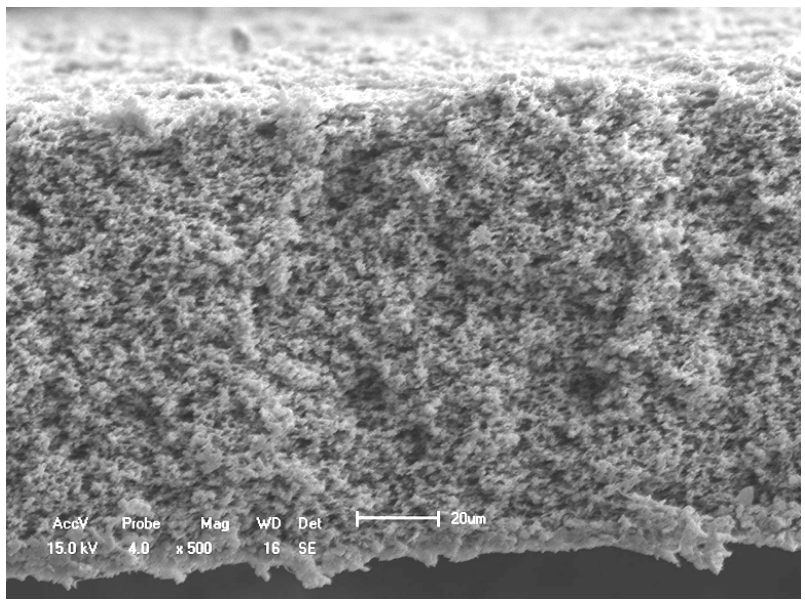

(d)

Figura 3. Micrografias (MEV) das membranas de PVDF: superfície (2000×) a) membrana A e b) membrana C; seção transversal (500x) c) membrana A e d) membrana $\mathrm{C}$.

matriz polimérica. A membrana comercial apresentou fluxo permeado igual à taxa de alimentação.

\section{Difusão de íons}

O coeficiente de difusão de íons para as membranas A e B estão apresentados na Figura 2. O coeficiente de difusão de íons decresce com o tempo, pode-se observar que a membrana com tratamento térmico apresentou coeficiente de difusão menor do que a membrana sem tratamento. Não foi possível verificar o coeficiente de difusão de íons para a membrana comercial que é altamente hidrofílica.

\section{Microscopia Eletrônica de Varredura (MEV)}

A morfologia das membranas é apresentada no Figura 3. Para a realização desta análise foi necessária a remoção do suporte, já 
Tabela 1. Dados obtidos a partir dos termogramas de DSC para o PVDF, o suporte de poliéster e para as membranas.

\begin{tabular}{|c|c|c|c|c|c|c|c|c|}
\hline & \multicolumn{4}{|c|}{ Fusão } & \multicolumn{4}{|c|}{ Cristalização } \\
\hline & \multicolumn{2}{|c|}{ PVDF } & \multicolumn{2}{|c|}{ Poliéster } & \multicolumn{2}{|c|}{ PVDF } & \multicolumn{2}{|c|}{ Poliéster } \\
\hline & $\Delta \mathbf{H}_{\mathrm{m}}\left(\mathrm{J} \cdot \mathrm{g}^{-1}\right)$ & $\mathbf{T}_{\mathrm{m}}\left({ }^{\circ} \mathbf{C}\right)$ & $\Delta \mathbf{H}_{\mathrm{m}}\left(\mathrm{J} \cdot \mathrm{g}^{-1}\right)$ & $\mathbf{T}_{\mathrm{m}}\left({ }^{\circ} \mathbf{C}\right)$ & $\Delta \mathbf{H}_{\mathrm{c}}\left(\mathrm{J}^{\prime} \mathrm{g}^{-1}\right)$ & $\mathbf{T}_{\mathrm{c}}\left({ }^{\circ} \mathbf{C}\right)$ & $\Delta \mathbf{H}_{\mathrm{c}}\left(\mathrm{J} \mathrm{g}^{-1}\right)$ & $\mathbf{T}_{\mathrm{c}}\left({ }^{\circ} \mathbf{C}\right)$ \\
\hline Poliéster & - & - & $-33,4$ & 248,7 & - & - & 31,2 & 211,4 \\
\hline Membrana A & $-18,1$ & 169,5 & $-27,4$ & 253,2 & 17,5 & 142,7 & 29,3 & 202,8 \\
\hline Membrana B & $-16,3$ & 169,5 & $-27,2$ & 253,2 & 15,8 & 142,8 & 28,5 & 208,5 \\
\hline Membrana C & $-40,7$ & 168,1 & - & - & 41,8 & 140,3 & - & - \\
\hline
\end{tabular}

que não foi possível fraturá-lo criogenicamente. Observa-se que a membrana A apresenta duas regiões durante a precipitação do polímero, uma região superior chamada de "pele" filtrante, que apresenta poros em torno de $350 \mathrm{~nm}$ que é a principal responsável por sua seletividade, e uma região porosa denominada suporte, que tem como principal função fornecer resistência mecânica à "pele" ${ }^{[10]}$. Em geral, a "pele" filtrante é obtida quando a separação líquido-líquido ocorre lentamente ${ }^{[1]}$.

As membranas A e B apresentaram a mesma morfologia, tanto na secção transversal como na superfície (com poros), sendo assim optou-se por apresentar somente as micrografias da membrana A. Já a membrana C é macroporosa inclusive com uma estrutura porosa ao longo da secção transversal muito semelhante às membranas A e B. Em ambos os casos tratam-se de membranas compósitas anisotrópicas porosas.

\section{Análise Termogravimétrica (TGA)}

As análises térmicas de TGA foram também realizadas para o PVDF, o suporte de poliéster e para as membranas, a fim de se estimar a perda de massa percentual e a temperatura em que ocorre a degradação da amostra, mostrado na Figura 4.

A Figura 4 apresenta as curvas de perda de massa onde foi possível verificar que a faixa de degradação do poliéster está entre 300 - $500{ }^{\circ} \mathrm{C}^{[6]}$, já para o PVDF se observa duas faixas de degradação, sendo a primeira em aproximadamente $180-350{ }^{\circ} \mathrm{C}$ e a segunda em torno de $360-550{ }^{\circ} \mathrm{C}^{[11]}$. O primeiro fenômeno observado para o PVDF pode estar relacionado a moléculas de baixo peso molecular, já que a temperatura tende a degradar, inicialmente, moléculas de baixo peso molecular ${ }^{[12]}$. Este primeiro fenômeno é atribuído à presença de plastificantes no polímero utilizado, o qual já havia sido informado pelo fabricante. $\mathrm{O}$ segundo fenômeno para o PVDF e as membranas é atribuído à degradação do polímero. De acordo com Campos et al. ${ }^{[13]}$, o PVDF sofre forte degradação acima de $400{ }^{\circ} \mathrm{C}$.

A degradação do PVDF das membranas A e B ocorreu em faixa de temperatura inferior à faixa observada para o PVDF puro e para a membrana C. Esta redução da temperatura está provavelmente relacionada ao poliéster. A massa residual segue o mesmo critério de proporção para as membranas em relação às fases. A membrana comercial apresentou uma massa residual superior às outras membranas e também superior a amostra de PVDF. Pode-se notar ainda que a membrana $\mathrm{C}$ não apresenta duas faixas de degradação, pois não contém plastificantes.

\section{Calorimetria exploratória diferencial (DSC)}

As análises térmicas de DSC foram realizadas para: PVDF, suporte de poliéster e membranas, a fim de estimar os valores da entalpia de fusão $\left(\Delta \mathrm{H}_{\mathrm{m}}\right)$, entalpia de cristalização $\left(\Delta \mathrm{H}_{c}\right)$, temperatura de fusão $\left(\mathrm{T}_{\mathrm{m}}\right)$ e cristalização $\left(\mathrm{T}_{\mathrm{c}}\right)$. Os resultados estão apresentados na Tabela 1 .

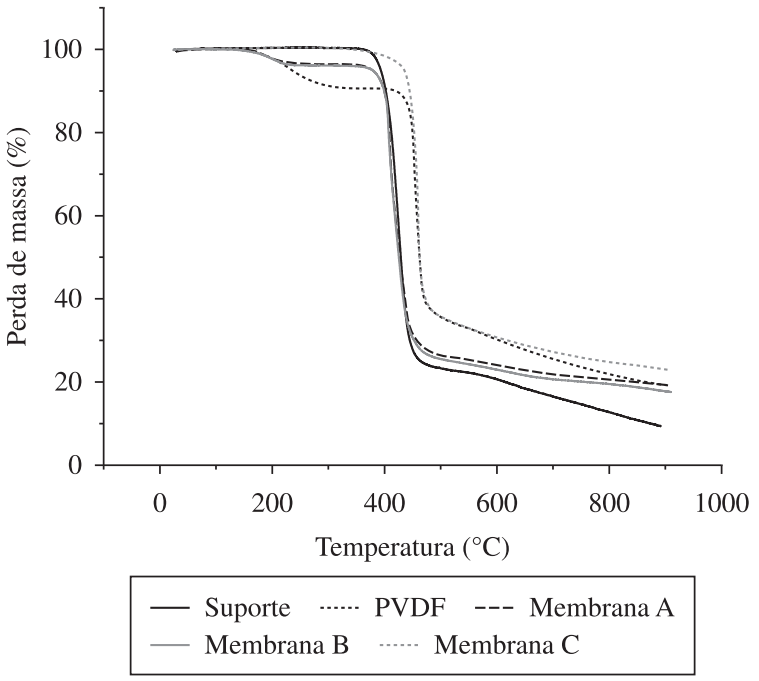

Figura 4. Termogramas de TGA das amostras de PVDF, suporte de poliéster e membranas.

$\mathrm{O}$ valor encontrado para $\Delta \mathrm{H}_{\mathrm{m}}$ do PVDF utilizado para o preparo das membranas é inferior ao descrito na literatura ${ }^{[13]} \mathrm{e}$ a Tm apresenta-se um pouco superior. Os valores encontrados para Tm e $\Delta \mathrm{H}_{\mathrm{m}}$, para o poliéster, estão coerentes aos descritos na literatura ${ }^{[6]}$. Relacionando os valores de ambas as fases com as membranas A e B, foi observado que o $\Delta \mathrm{H}_{\mathrm{m}}$ de ambas as fases apresentou redução, sendo possivelmente uma função da relação PVDF/Poliéster. Também na Tabela 1, pode-se notar que os valores de $\Delta \mathrm{H}_{\mathrm{c}}$ estão concordantes com os de $\Delta \mathrm{H}_{\mathrm{m}}$. A redução de entalpia de cristalização, assim como a de fusão, estão relacionadas, possivelmente, a reorganização conformacional da estrutura de ambas as fases ${ }^{[12]}$. A membrana $\mathrm{C}$ apresentou $\Delta \mathrm{H}_{\mathrm{m}}$ e $\Delta \mathrm{H}_{\mathrm{c}}$ superiores aos valores encontrados para $\mathrm{o}$ PVDF utilizado na fabricação das membranas A e B, fato que pode ser justificado pela ausência de plastificante na membrana $\mathrm{C}$.

\section{Conclusão}

Com base nos resultados obtidos na pressão de 6 bar, podemos concluir que as membranas A e B apresentam resistência maior à passagem de água, ou seja, apresentam fluxo de água permeada menor do que a membrana $\mathrm{C}$. Isso também se deve ao fato das membranas desenvolvidas apresentarem tamanho e quantidade de poros menores em relação à membrana comercial, ou seja, as membranas desenvolvidas apresentam a "pele" filtrante, o que não aparece na membrana comercial. $\mathrm{O}$ tratamento térmico realizado mostrou-se pouco eficaz, já que não obteve-se o resultado esperado em relação ao fluxo de água permeado e à morfologia da superfície da membrana B. Sendo assim, temperaturas superiores devem ser utilizadas para realização do tratamento térmico em membranas de PVDF. 


\section{Agradecimentos}

Os autores agradecem ao CNPq, CAPES e Hidrofiltros do Brasil Ltda. pelos auxílios e bolsas ao projeto.

\section{Referências Bibliográficas}

1. Habert, A. C.; Borges, C. P. \& Nobrega, R. - "Processos de separação com membranas", E-papers, Rio de Janeiro, 2006.

2. Hamza, A.; Pham, V. A.; Matsuura, T. \& Santerre, J. P. - J. Membr. Sci., 131, p.217 (1997).

3. Loeb, S. \& Sourirajan, S. - UCLA Dept of Eng Report (1960).

4. Silva, H. L. B. - "Uso de membranas microporosas no tratamento de efluentes de um frigorífico de abate de aves", Dissertação de Mestrado, Universidade Federal de Santa Catarina, Brasil (2005).

5. Bottino, A.; Capannelli, G.; Monticelli, O. \& Piaggio,P. - J. Membr. Sci., 166, p.23 (2000).

6. Mark, J. E. - "Polymer Data Handbook", Oxford University Press, New York (1999).

7. Carvalho, R. B.; Borges, C. P. \& Nóbrega, R. - Polímeros, 11, p.65 (2001).
8. Chang, Y.; Shilh, Y.; Ruaan, R.; Higuchi, A.; Chen, W. \& Lai, J. J Membr Sci, 309, p.165 (2008).

9. Meireles, C. S.; Filho, G. R.; Assunção, R. M. N.; Cerqueira, D. A.; Zeni, M.; Mello, K. \& Lorenzi, S. - Polym. Eng. Sci., 48, p.1443 (2008).

10. Zeni, M.; Riveros, R.; Mello, K. \& Duarte, J. - Desaliantion, 221, p.294 (2008).

11. Zucolotto, V.; Filho, R. G.; Avlyanov, J.; Mattoso, L. H. C. - Polímeros, 12, p.213 (2002).

12. Lucas, E. F.; Soares, B. G. \& Monteiro, E. - "Caracterização de Polímeros: Determinação de Peso Molecular e Análise Térmica", E-papers, Rio de Janeiro (2001).

13. Campos, J. S. C.; Ribeiro, A. A. \& Cardoso, C. X. - Mater. Sci. Eng. B, 136, p.123 (2007).

Enviado: 30/10/09

Reenviado: $21 / 01 / 10$

Aceito: $11 / 02 / 10$

DOI: $10.1590 / \mathrm{S} 0104-14282010005000037$ 